\title{
Associated risk factors of lower limb lymphedema after treatment of cervical and endometrial cancer
}

\author{
LAURA FLORENTINA REBEGEA ${ }^{1,2^{*}}$, GABRIELA STOLERIU $^{2}$, NICUTA MANOLACHE $^{3}$, CRISTINA SERBAN $^{4,5}$, \\ MIHAELA CRAESCU ${ }^{1,6}$, MARY-NICOLETA LUPU ${ }^{5,7^{*}}$, DOINA CARINA VOINESCU ${ }^{2 *}$, \\ DOREL FIRESCU ${ }^{4,5}$ and OANA ROXANA CIOBOTARU ${ }^{2}$
}

\author{
${ }^{1}$ Department of Radiotherapy and Oncology, 'St. Apostle Andrew' County Emergency Clinical Hospital, \\ 800179 Galați; ${ }^{2}$ Clinical Department and ${ }^{3}$ Department of Pharmaceutical Sciences, Faculty of Medicine and Pharmacy, \\ 'Dunarea de Jos' University, 800008 Galati; ${ }^{4}$ Second Surgery Clinic, 'St. Apostle Andrew' County Emergency Clinical Hospital, \\ 800179 Galati; Departments of ${ }^{5}$ Surgery and ${ }^{6}$ Morphological and Functional Sciences, Faculty of Medicine \\ and Pharmacy, 'Dunarea de Jos' University, 800008 Galati; ${ }^{7}$ Anaesthesia and Intensive Care Clinic, \\ ‘St. Apostle Andrew’ County Emergency Clinical Hospital, 800179 Galați, Romania
}

Received May 5, 2020; Accepted June 4, 2020

DOI: $10.3892 / e t m .2020 .9311$

\begin{abstract}
The scope of the study was to identify the associated risk factors of lower limb lymphedema development in cervical and endometrial cancer patients. We retrospectively analysed 326 patients: 186 cases $(57.06 \%)$ with cervical cancer and 140 cases (42.94\%) with endometrial cancer were treated in Surgery, Radiotherapy, Oncology and Gynaecology Clinics of 'St. Apostle Andrew' Emergency Clinical Hospital Galati over 9 years. Adjuvant radiotherapy was performed in $83.57 \%$ of endometrial cancer cases. Adjuvant chemotherapy was
\end{abstract}

Correspondence to: Professor Gabriela Stoleriu, Clinical Department, Faculty of Medicine and Pharmacy, 'Dunarea de Jos' University, 35 Al. I. Cuza Street, 800008 Galati, Romania

E-mail: stoleriugabriela@yahoo.com

Dr Cristina Serban, Department of Surgery, Faculty of Medicine and Pharmacy, 'Dunarea de Jos' University, 35 Al. I. Cuza Street, 800008 Galati, Romania

E-mail: cristina_g186@yahoo.com

${ }^{*}$ Contributed equally

Abbreviations: 95\% CI, confidence interval; BMI, body mass index; BSO, bilateral salpingo-oophorectomy; BT, brachytherapy; CC, cervical cancer; CMT, chemotherapy; CT, computed tomography scan; EC, endometrial cancer; HR, hazard ratio; LA, lymphadenectomy; LLL, lower limb lymphedema; NSAIDs, nonsteroidal antiinflammatory drugs; OR, odds ratio; $\mathrm{p}$, PCC, Pearson correlation coefficient; PALA, para-aortic lymphadenectomy; PLA, pelvic lymphadenectomy; RT, radiotherapy; S, surgery; TAH, total abdominal hysterectomy; vs., versus

Key words: gynaecological cancers, endometrial cancer, cervical cancer, lymphedema, risk factors performed in $45.16 \%$ of cervical cancer cases. Over 10 lymph nodes were removed in $74.73 \%$ of cervical cancer patients. Incidence of lymphedema was $15.05 \%$ in cervical cancer patients and $10 \%$ in endometrial cancer patients, $\mathrm{P}=0.06$. Analysed risk factors for lower limb lymphedema occurrence were: Age, disease stage, radiotherapy, number of invaded lymph nodes (for cervical cancer patients), number of removed lymph nodes (for cervical cancer patients) and obesity. Multivariate analysis for associated risk factors of lower limb lymphedema development in cervical cancer showed that number of removed lymph nodes, OR=2.109 (0.907-4.903), $\mathrm{P}<0.0001$, number of lymph nodes with metastasis, $\mathrm{OR}=1.903$ (0.253-4.332), $\mathrm{P}=0.004$ and obesity, $\mathrm{OR}=1.713$ (0.226-2.967), $\mathrm{P}=0.006$ were found as statistically significant risk factors for lower limb lymphedema onset. For endometrial cancer patients, obesity, $\mathrm{OR}=1.518(0.721-2.75), \mathrm{P}=0.0003$, was the only associated risk factor with statistical significance for the lower limb lymphedema development. Lower limb lymphedema represents one of the adverse reactions of multimodal treatment in gynaecological cancers which affects patient's quality of life. Lower limb lymphedema occurrence is related with number of risk factors, the most important being removed lymph nodes, obesity and radiotherapy.

\section{Introduction}

Gynaecological cancers represent $16.3 \%$ of total malignant diseases, cervical cancer (CC) and endometrial cancer (EC) being the most common. The main therapeutic options are surgery (S) with pelvic lymphadenectomy (PLA) with or without para-aortic lymphadenectomy (PALA) and chemotherapy (CMT), external beam radiotherapy (RT), brachytherapy (BT). Lymphadenectomy with or without PALA is used in different stages. The association PLA with PALA increases the probability of adverse reactions.

Lower limb lymphedema (LLL) represents one of most debilitating adverse reactions occurring after multimodal 
treatment of gynaecological cancers, surgery and radiotherapy. For many cases of gynaecological neoplasia, lymphadenectomy represents a part of the staging being in integral part of surgery, and this procedure is associated with LLL. In literature, incidence of lymphedema varies in very large limits, 1-67\% after multimodal treatment (S, RT, CMT). These very large limits are due to lack of common criteria for lymphedema evaluation. Specialty literature uses many ways to define lymphedema, in arbitrary mode, from non-symptomatic to symptomatic lymphedema. The first published study trying to define lymphedema was by Kim et al (1) published in 2017. In this study the computed tomography scan (CT) for abdomen and pelvic areas was used as screening test for LLL occurrence (1). Among risk factors mentioned for LLL occurrence are number of removed lymph nodes, iliac lymph node excision, and adjuvant radiotherapy.

The present aimed to identify the risk factors associated with LLL occurrence after multimodal treatment for patients with cervical cancer and EC.

In general, lymphedema is caused by obstruction or interruption of lymphatic drainage, in distal segment, due to infections and malignancies. LLL represents a chronic complication and can lead to physical and psychological suffering $(1,2)$ being one of the most important causes of poor quality of life in patients with gynaecologic malignancies after multimodal treatment (1). Lymphedema can be complicated by inflammation, infection, local skin or mucous membrane changes (including microbiome changes). The drugs used for treatment of lymphedema (systemic diuretics, tonic drugs for lymphatic vessels), AINS, or topical treatments $(3,4)$ or for its complication (infections, comorbidities, pain) are also sometimes the source of adverse reactions with associated poor quality of life $(5,6)$.

\section{Patients and methods}

This is a retrospective study with 326 patients, $186(57.06 \%)$ with CC and 140 (42.94\%) with EC treated in the Surgery, Radiotherapy, Oncology, Gynaecology Departments of 'St. Apostle Andrew' Emergency Clinical Hospital (Galați, Romania), between 01/01/2007 and 31/12/2015. The study was approved by the Ethics Committee of 'St. Apostle Andrew' County Emergency Clinical Hospital. Informed consent was obtained from each patient prior to investigations, treatment and participation in the study. Patients with stage IIIB or IV disease, with other synchronous neoplasia, who presented lymphedema before surgery for pelvic cancer, with secondary neoplastic lesions, with profound venous thrombosis or who did not present at follow-up medical visits were excluded from study. Informed consent was obtained for each patient $(7,8)$. The median follow-up period was 54 months (range 12-108 months).

Patients with lower limb volume increase $>5 \%$ compared with the other lower limb were considered to have lymphedema. Clinical and therapeutic parameters are showed in Tables I and II, respectively.

Statistical analysis. Patient characteristics were compared using parametrical test, Student's t-test, Z-test (function of group size, for mean comparison) and non-parametrical
Mann-Whitney U test (for median comparison) (Table I). Multivariate logistic regression analysis was used to assess the risk factors associated as independent for occurrence of post-treatment LLL. Pearson's correlation coefficient (PCC) was used for bivariate correlation test. Statistical significance was set at $\mathrm{P} \leq 0.05$. The statistical calculations were performed using XL-STAT software.

\section{Results}

Median age was 53.5 years in CC group vs. 63 years in EC group $(\mathrm{P}<0.0001)$. Stage IIB of disease predominated in the CC group (78.49\% of cases) and in EC group, the patients with stage II disease were more frequent ( $83.57 \%$ of cases). Squamous carcinoma was the most frequent histology in CC $(82.8 \%$ of patients) vs. adenocarcinoma in EC $(89.29 \%$ of cases), $\mathrm{P}<0.0001$. Tumor grade $\mathrm{G} 2$ predominated in both groups. Obesity was found in $10.22 \%$ of patients in $\mathrm{CC}$ and in $28.57 \%$ of cases in $\mathrm{EC}, \mathrm{P}=0.06$.

Patient characteristics were compared using parametrical test, Student's t-test, Z-test (function of group size, for mean comparison) and non-parametrical Mann-Whitney U test (for median comparison) (Table I).

All CC patients underwent surgery, total abdominal hysterectomy (TAH) with bilateral salpingo-oophorectomy (BSO) and lymphadenectomy (LA). All EC patients underwent surgery, TAH with BSO without LA. Adjuvant RT was performed in $23.66 \%$ of CC patients and $83.57 \%$ of EC patients. BT was administered to $96.24 \%$ of CC patients and $16.43 \%$ of EC patients. None of EC patients received CMT and in CC group, adjuvant CMT was performed in $45.16 \%$ of the cases. In $\mathrm{CC}, 74.73 \%$ of cases had $\geq 10$ lymph nodes removed and $25.27 \%$ of cases had $<10$ lymph nodes removed. In this group, $88.71 \%$ of patients had $<10$ lymph node metastasis and only 21 patients $(11.29 \%)$ had $\geq 10$ lymph node metastasis (Table II).

Comorbidities are presented in Table III. High blood pressure was the most frequent associated comorbidity found in $13.98 \%$ of CC patients and in $49.29 \%$ of EC patients. Obesity was present in $28.57 \%$ of EC cases and in $10.22 \%$ of CC cases. Other frequently associated comorbities were diabetes mellitus type II in $24.29 \%$ of EC cases and secondary anaemia in $26.34 \%$ of CC patients. Coronary disease was finding in $9.14 \%$ of CC cases. Two CC patients were pregnant at the time of diagnosis (9).

The incidence of LLL was $15.05 \%$ of CC patients and $10 \%$ of $\mathrm{EC}$ cases, $\mathrm{P}=0.06$. LLL was unilateral in all patients enrolled in this study. In cervical cancer patients, $55.9 \%$ had left LLL and $44.09 \%$ had right LLL; the median time of LLL occurrence post-surgery was 4.5 months (range 2-18 months). In the EC group, $54.29 \%$ had left LLL and $45.71 \%$ had right LLL; the median time of LLL post-surgery occurrence was 2 months (range 1-4 months).

Univariate analysis for associated risk factors of LLL development in $\mathrm{CC}$ revealed that age under 50 years $(\mathrm{P}=0.05)$, neoadjuvant $\mathrm{RT}(\mathrm{P}<0.001)$ and $\geq 10$ lymph nodes with metastasis $(\mathrm{P}=0.05)$ were found to be the predictive risk factors with statistical significance (Table IV). Due to the limit of significance level $(\mathrm{P}=0.05)$ some risk factors such as age, number of lymph node metastasis from univariate analysis, we performed also multivariate analysis for these parameters. 
Table I. Clinical parameters.

\begin{tabular}{lccr}
\hline Parameter & $\begin{array}{c}\text { Cervical cancer } \\
\mathrm{N}(\%)\end{array}$ & $\begin{array}{c}\text { Endometrial cancer } \\
\mathrm{N}(\%)\end{array}$ & P-value \\
\hline Median age (years) & $53.5(24-83)$ & $63(27-82)$ & $<0.0001$ \\
TNM stage & - & $9(6.43)$ & 0.703 \\
IA & $30(16.13)$ & $14(10)$ & \\
IB & $10(5.38)$ & Stage II: $117(83.57)$ & $<0.0001$ \\
IIA & $146(78.49)$ & & \\
IIB & & & \\
Histopathology & $154(82.8)$ & $125(89.29)$ & $7(5)$ \\
Squamous carcinoma & $30(16.13)$ & $1(0.72)$ & \\
Adenocarcinoma & $2(1.08)$ & $5(3.57)$ & \\
Adenosquamous carcinoma & 0 & & \\
Leiomyosarcoma & 0 & $38(27.14)$ & \\
Fibrosarcoma & & $75(53.57)$ & 0.54 \\
Grading & $33(17.74)$ & $27(19.29)$ & 0.06 \\
G1 & $59(31.72)$ & $40(28.57)$ & \\
G2 & $94(50.54)$ & & \\
G3 & $19(10.22)$ & & \\
Obesity & & & \\
\hline
\end{tabular}

$\mathrm{N}$, number of cases.

Univariate analysis for associated risk factors of LLL development in EC group revealed that age $>50$ years $(\mathrm{P}<0.001)$, adjuvant $\mathrm{RT}(\mathrm{P}<0.001)$ and obesity $(\mathrm{P}=0.001)$ were found to be the predictive risk factors with statistical significance for LLL occurrence (Table V).

For patients with EC, LA was not performed, and consequently, the number of lymph nodes retrieved and numbers of metastatic lymph nodes were not analysed as independent risk factors.

Multivariate analysis for associated risk factors of LLL development in CC showed that number of removed lymph nodes, $\mathrm{OR}=2.109$ (0.907-4.903), $\mathrm{P}<0.0001$, number of lymph nodes with metastasis, $\mathrm{OR}=1.903(0.253-4.332), \mathrm{P}=0.004$ and obesity, $\mathrm{OR}=1.713(0.226-2.967), \mathrm{P}=0.006$ were found as statistically significant risk factors for LLL occurrence. The analytical method used was multivariate logistic regression. For EC patients, obesity, OR=1.518 (0.721-2.75), $\mathrm{P}=0.0003$, was the only associated risk factor with statistical significance for LLL development (Table VI).

PCC for LLL indicates that the strongest correlation was found between LLL occurrence and number of removed lymph nodes for CC (PCC=0.713). The PCC values for CC cases also indicate a correlation between LLL occurrence and number of lymph nodes with metastasis, $\mathrm{PCC}=0.368$ and obesity, $\mathrm{PCC}=0.163$. In the EC group PCC values indicate strong correlation between obesity and LLL occurrence, PCC $=0.689$ (Table VII).

Comparative analysis of patients who developed LLL with cervical cancer vs. EC revealed that the only parameter that significantly varied was neoadjuvant RT, performed in
$17.86 \%$ of CC cases with LLL vs. $100 \%$ of EC cases with LLL, $\mathrm{P}=0.01$.

\section{Discussion}

LLL represents one of the adverse reactions of multimodal treatment of gynaecological cancers (S, RT, CMT) which have a negative impact on quality of life. LLL has less known characteristics (time of occurrence, duration and evolution), is caused by a disruption/obstruction of the lymphatic system, in general, in proximal segment of thigh and is due to neoplasia and infections (10).

For many gynaecological malignancies, LA represents an integral part of staging and treatment and this procedure was associated with LLL occurrence. LLL incidence varies in very large limits in specialty literature, and is influenced by the gynaecological cancer sites; the highest incidence is in vulvar cancer and the lowest, in ovarian cancer (10).

A study by Ki et al (11), indicated the prevalence of LLL in cancer patients, function of anatomic origins: $9-70 \%$ in vulvar cancer, $1.2-47 \%$ in cervical cancer, $1.2-17.7 \%$ in EC and $7-40.8 \%$ in ovarian cancer.

The present study shows the involved risk factors of arm lymphedema occurring after breast cancer treatment are similar with risk factors which influence the LLL development after pelvic malignancies (12). These risk factors could be the number of removed lymph nodes and adjuvant radiotherapy.

A concrete method for lymphedema diagnosis has not yet been establish. The physical examination performed by an experienced physician is the most common diagnostic 
Table II. Therapeutic parameters.

Treatment performed

total number of patients,

$\mathrm{N}=326$
Cervical cancer, N (\%)

$\mathrm{N}=186(57.06 \%)$
Endometrial cancer, N (\%) $\mathrm{N}=140(42.94 \%)$

\section{Surgery}

$\mathrm{TAH}+\mathrm{BSO}+\mathrm{LA}$

$186(100)$

$142(76.34)$

$44(23.66)$

$179(96.24)$

$84(45.16)$

0

Neoadjuvant

Number of removed lymph nodes

$<10$

$\geq 10$

Number of lymph nodes with metastasis

$<10$

$\geq 10$
47 (25.27)

139 (74.73)

$165(88.71)$

$21(11.29)$
140 (100) (without LA)

0

$117(83.57)$

$23(16.43)$

0

0

0

TAH, total abdominal hysterectomy; BSO, bilateral salpingo-oophorectomy; LA, lymphadenectomy; BT, brachytherapy; N, number of cases .

Table III. Associated comorbidities.

\begin{tabular}{lcc}
\hline $\begin{array}{l}\text { Associated } \\
\text { comorbidities }\end{array}$ & $\begin{array}{c}\text { Cervical } \\
\text { cancer } \\
\mathrm{N}=186(\%)\end{array}$ & $\begin{array}{c}\text { Endometrial } \\
\text { cancer } \\
\mathrm{N}=140(\%)\end{array}$ \\
\hline High blood pressure & $26(13.98)$ & $69(49.29)$ \\
Diabetes mellitus type II & $8(4.3)$ & $34(24.29)$ \\
Obesity & $19(10.22)$ & $40(28.57)$ \\
Hepatitis & $9(4.84)$ & $11(7.86)$ \\
Cirrhosis & $2(1.08)$ & 0 \\
Renal lithiasis & $2(1.08)$ & 0 \\
Biliary lithiasis & $2(1.08)$ & $4(2.86)$ \\
Pulmonary tuberculosis & $2(1.08)$ & 0 \\
Thyroid diseases & $3(1.61)$ & $2(1.43)$ \\
Anxious depressive syndrome & $2(1.08)$ & $1(0.71)$ \\
Coronary disease & $17(9.14)$ & $7(4.29)$ \\
Pregnancy & $2(1.08)$ & 0 \\
\hline
\end{tabular}

$\mathrm{N}$, number of cases.

method. A screening method for early LLL detection is not yet established. Kim et al (1) was the first to use computed tomography scan (CT) as screening instrument for LLL risk factor identification in patients who underwent surgery for LA pelvic neoplasia. In that study, 511 patients with pelvic malignancies (cervical, uterine, ovarian and vulvar cancers) were analysed. Of those patients, $106(20.7 \%)$ were diagnosed with LLL. The results of their study indicate that the risk factors for LLL occurrence after LA in gynaecological cancers could be the number of lymph nodes retrieved (median $>30$ ), adjuvant pelvic radiotherapy $(\mathrm{P}<0.001)$, surgical intervention (open surgery or laparoscopy), long operation time, not using of intermittent pneumatic compression (1). Criteria for diagnosis or screening of LLL by imaging tests have not yet been established. The authors used, in arbitrarily way, the mean difference of subcutaneous layer thicknesses between preoperative and postoperative (after 1 year) CT scans, for the 106 patients with LLL. The authors measured the subcutaneous layer thicknesses over the Sartorius muscle of the right anterior thigh of the level of lesser trochanter of femur (1). The reference value was $3.77 \pm 3.14 \mathrm{~mm}$ and value was used as grouping criteria of patients.

In a study performed by Kim et al (13), adjuvant RT was significant associated with LLL lymphedema in patients with stage IIA FIGO cervical cancer, who performed radical surgery with LA. Their study enrolled 707 patients, and $12.6 \%$ of cases developed LLL. Number of resected lymph nodes was not found as a risk factor with statistical significance for LLL occurrence. Adjuvant RT was found a predictive risk factor for LLL development (OR=3.47; 95\% CI, 2.086-5.788). Their study also concluded that patients need to be informed before treatment on the possibility of LLL occurrence and on the ways to prevent it (13).

In previous studies, risk factor analysis for LLL incidence identified that pelvic LA is a statistically significant factor, after the malignant diagnosis, which can interrupt the lymphatic flow (14-17).

In the study of Tada et al (14), 694 patients with cervical, ovarian and ECs were analysed. Among uterine and ovarian cancer patients who underwent pelvic LA and post-operative radiotherapy $(\mathrm{OR}=1.79 ; 95 \% \mathrm{CI}, 1.20-2.67 ; \mathrm{P}=0.006)$ was statistically significantly associated with LLL development. 
Table IV. Univariate analysis for associated risk factors of LLL development in CC group (186 cases).

\begin{tabular}{lcc}
\hline & Non lymphedema & Lymphedema \\
Analyzed parameter & $158(84.95 \%)$ & $28(15.05 \%)$ \\
\hline Age (years) & & $19(67.86)$ \\
$\leq 50$ & $62(39.24)$ & $9(32.14)$ \\
$>50$ & $96(60.76)$ & $23(82.14)$ \\
Neoadjuvant radiotherapy $(\mathrm{N}=142)$ & $119(75.32)$ & $5(17.86)$ \\
Adjuvant radiotherapy $(\mathrm{N}=44)$ & $39(24.68)$ & $9(32.14)$ \\
Number of removed lymph nodes & $38(24.05)$ & $19(67.86)$ \\
$<10$ & $120(75.95)$ & 0.57 \\
$\geq 10$ & & $20(71.42)$ \\
Number of lymph nodes with metastasis & $145(91.77)$ & $8(28.57)$ \\
$<10$ & $13(8.23)$ & 0.06 \\
\hline 10 & & 0.05 \\
\hline
\end{tabular}

LLL, lower limb lymphedema; CC, cervical cancer; N, number of cases.

Table V. Univariate analysis for associated risk factors of LLL development in EC group (140 cases).

\begin{tabular}{lccc}
\hline Analyzed parameter & $\begin{array}{c}\text { Non lymphedema } \\
\mathrm{N}=126(90 \%)\end{array}$ & $\begin{array}{c}\text { Lymphedema } \\
\mathrm{N}=14(10 \%)\end{array}$ & \\
\hline Age (years) & & 0 & $<0.001$ \\
$\leq 50$ & 8 & $14(100)$ & $11(78.57)$ \\
$>50$ & 118 & $14(100)$ & 0.001 \\
Obesity & $29(23.02)$ & & $<0.001$ \\
Adjuvant radiotherapy & $103(81.75)$ & & \\
\hline
\end{tabular}

LLL, lower limb lymphedema; EC, endometrial cancer; N, number of cases.

Table VI. Multivariate analysis for associated risk factors of LLL development.

\begin{tabular}{llr}
\hline Risk factors & OR (95\% CI) & P-value \\
\hline Cervical cancer & & $<0.0001$ \\
Number of removed lymph nodes & $2.109(0.907-4.903)$ & 0.004 \\
Number of lymph nodes with metastasis & $1.903(0.253-4.332)$ & 0.006 \\
Obesity & $1.713(0.226-2.967)$ & 0.0003 \\
Endometrial cancer & $1.518(0.721-2.75)$ & \\
Obesity & & 0.006 \\
\hline
\end{tabular}

LLL, lower limb lymphedema; N, number of cases.

Table VII. Pearson's correlation coefficient.

\begin{tabular}{lcc}
\hline & \multicolumn{2}{c}{ Pearson's correlation coefficient } \\
\cline { 2 - 3 } Correlated parameters & Cervical cancer & Endometrial cancer \\
\hline Number of removed lymph nodes & 0.713 & - \\
Number of lymph nodes with metastasis & 0.368 & - \\
Obesity & 0.163 & 0.689 \\
\hline
\end{tabular}

$\mathrm{N}$, number of cases. 
Pelvic LA was not found as a risk factor for occurrence of lymphedema.

A study by Abu-Rustum et al (18) identified $\geq 10$ resected lymph nodes during surgery for EC, being a risk factor for development of LLL. Their retrospective study was conducted over a period of 12 years and analysed 1,289 patients with EC. LLL occurred 5.3 months after surgery (range 1-32 months). Median follow-up period was 3 years. Age, weight, type of surgery, type of adjuvant RT and stage were not associated with LLL occurrence. Patients who had $>10$ regional lymph nodes resected seemed to be at most significant risk for occurrence of leg lymphedema, after surgery for EC.

Kuroda et al (19) retrospectively analysed 264 patients with gynaecologic malignancies. LLL developed after a median period of 13.5 months. Regression analysis revealed that body mass index, $\mathrm{BMI} \geq 25 \mathrm{~kg} / \mathrm{m}^{2} \quad(\mathrm{HR}=1.616$; 95\% CI, 1.030-2.535), pelvic LA with PALA (HR=2.323; 95\% CI, 1.126-4.794), adjuvant RT (HR=2.469; 95\% CI, 1.148-5.310) and lymphocyte formation ( $\mathrm{HR}=1.718 ; 95 \% \mathrm{CI}$, 1.120-2.635), were independently associated with LLL occurrence. The data from their study showed that the occurrence rate of LLL increases logarithmically, from $32.8 \%$ at 3 years to $47.7 \%$ at 10 years.

In 2011, Ohba et al (20) published an article which analysed risk factors for lower-limb lymphedema after surgery for cervical cancer. A total of 155 patients with cervical cancer were evaluated with a median follow-up of 6.1 years. Thirty-one patients $(20.0 \%)$ developed LLL. Supra-femoral node dissection $(\mathrm{OR}=9.5$; $95 \% \mathrm{CI}, 1.2-73.3 ; \mathrm{P}=0.031)$ and adjuvant $\mathrm{RT}(\mathrm{OR}=3.7 ; 95 \% \mathrm{CI}, 1.2-10.9 ; \mathrm{P}=0.019)$ were found to be independent risk factors).

Regarding risk factors for LLL occurring after treatment of pelvic malignancy, there are contradictory data.

There are some studies which identify that number of resected lymph nodes was not found as an independent risk factor for LLL occurrence, such as the study of Tada et al (14) and of Kim et al (13).

There are studies which, contrarily, identified that number of resected lymph nodes was an independent risk factor for LLL occurrence $(18,21)$.

In the present study, multivariate analysis identified for cervical cancer group, risk factors associated with development of lymphedema as the number of resected lymph nodes $(\mathrm{P}<0.0001)$, number of lymph nodes with metastasis $(\mathrm{P}=0.004)$, obesity $(\mathrm{P}=0.006)$ and for $\mathrm{EC}$, the only statically-significant risk factor was obesity $(\mathrm{P}=0.0003)$.

LLL is a chronic, progressive and incurable condition which often leads to disability. A recent study by Mirandola et al (22) showed an improvement in lower limb swelling with ameliorated quality of life after the overall physical activity pathway. Cancer is a chronic disease that induces important changes to the functional capabilities of the patients, the body image and the social or family role of the patients causing further emotional problems. Therefore, the role of a multidisciplinary team is crucial in the management of the patients (23).

In conclusion, LLL represents an adverse reaction of multimodal treatment for pelvic neoplasia, affects the quality of life for women treated for gynaecology cancers and all patients must be informed, before treatment initiation, on the possibility of LLL occurrence after the treatment and on the prevention methods. The incidence of LLL is related with numerous risk factors, with the most important being the number of removed lymph nodes, obesity and adjuvant radiotherapy.

Limitation of our study is the retrospective aspect, thus there are no survival studies.

\section{Acknowledgements}

Not applicable.

\section{Funding}

No funding was received.

\section{Availability of data and materials}

The datasets used and/or analysed during the current study are available from the corresponding author on reasonable request.

\section{Authors' contributions}

LFR and ORC contributed to the design of the study, participated in the entire review process and prepared the manuscript. DCV, MNL, DF and NM contributed to the literature research, and the analysis and critical interpretation of the data. GS, CS and $\mathrm{MC}$ conceived the study and revised the manuscript. All authors read and approved the final version of the manuscript.

\section{Ethics approval and consent to participate}

The study was approved by the Ethics Committee of 'St. Apostle Andrew' County Emergency Clinical Hospital (Galați, Romania). Informed consent was obtained from each patient prior to investigations, treatment and participation in the study.

\section{Patient consent for publication}

Not applicable.

\section{Competing interests}

All the authors declare that they have no competing interests.

\section{References}

1. Kim M, Suh DH, Yang EJ, Lim MC, Choi JY, Kim K, No JH and Kim YB: Identifying risk factors for occult lower extremity lymphedema using computed tomography in patients undergoing lymphadenectomy for gynecologic cancers. Gynecol Oncol 144: 153-158, 2017.

2. Filip-Ciubotaru F, Manciuc C, Stoleriu G and Foia L: NADPH Oxidase: Structure and activation mecanisms (Review). Note I. Rev Med Chir Soc Med Nat Iasi 120: 29-33, 2016.

3. Ciobotaru OR, Lupu MN, Rebegea L, Ciobotaru OC, Duca OM, Tatu AL, Voinescu CD, Stoleriu G, Earar K and Miulescu M: Dexamethasone-chemical structure and mechanisms of action in prophylaxis of postoperative side effects. Rev Chim (Bucharest) 70: 843-847, 2019 (In Romanian).

4. Tatu AL, Ciobotaru OR, Miulescu M, Buzia OD, Elisei AM, Mardare N, Diaconu C, Robu S and Nwabudike LC: Hydrochlorothiazide: Chemical structure, therapeutic, phototoxic and carcinogenetic effects in dermatology. Rev Chim (Bucharest) 69: 2110-2114, 2018 (In Romanian). 
5. Nwabudike LC and Tatu AL: Response to - chronic exposure to tetracyclines and subsequent diagnosis for non-melanoma skin cancer in a large Mid-Western US population. J Eur Acad Dermatol Venereol 32: e159, 2018.

6. Brănişteanu DE, Pintilie A, Andreș LE, Dimitriu A, Oanţă A, Stoleriu G and Brănișteanu DC: Ethiopatogenic hypotheses in lichen planus. Rev Med Chir Soc Med Nat Iasi 120: 760-767, 2016.

7. Ciobotaru OR, Voinescu DC, Ciobotaru OC, Voicu D and Arbune M: Expression of p53 and Ki-67 in distal oesophageal and gastric cardia adenocarcinomas. Rom Biotechnol Lett 20: 10800-10808, 2015.

8. Voinescu DC, Ciobotaru OR, Ciobotaru OC, Preda A, Lupu VV, Coman MB and Arbune M: Ultrastructural changes of the gastric mucosa induced by the Helicobacter pylori infection. Rev Chim 66: 2104-2108, 2015.

9. Schaas BA, Ivan S, Titianu M, Condratovici CP, Maier A and Schaas CM: Biochemical markers predicting the risk of gestational diabetes mellitus. Mater Plast 54: 133-136, 2017.

10. Biglia N, Librino A, Ottino MC, Panuccio E, Daniele A and Chahin A: Lower limb lymphedema and neurological complications after lymphadenectomy for gynecological cancer. Int J Gynecol Cancer 25: 521-525, 2015.

11. Ki EY, Park JS, Lee KH and Hur SY: Incidence and risk factors of lower extremity lymphedema after gynecologic surgery in ovarian cancer. Int J Gynecol Cancer 26: 1327-1332, 2016.

12. Rebegea L, Firescu D, Dumitru M and Anghel R: The incidence and risk factors for occurrence of arm lymphedema after treatment of breast cancer. Chirurgia (Bucur) 110: 33-37, 2015.

13. Kim JH, Choi JH, Ki EY, Lee SJ, Yoon JH, Lee KH, Park TC Park JS, Bae SN and Hur SY: Incidence and risk factors of lower-extremity lymphedema after radical surgery with or without adjuvant radiotherapy in patients with FIGO stage I to stage IIA cervical cancer. Int J Gynecol Cancer 22: 686-691, 2012.

14. Tada H, Teramukai S, Fukushima M and Sasaki H: Risk factors for lower limb lymphedema after lymph node dissection in patients with ovarian and uterine carcinoma. BMC Cancer 9: 47, 2009.
15. Logmans A, Kruyt RH, de Bruin HG, Cox PH, Pillay M and Trimbos JB: Lymphedema and lymphocysts following lymphadenectomy may be prevented by omentoplasty: A pilot study. Gynecol Oncol 75: 323-327, 1999.

16. Ilancheran A and Monaghan JM: Pelvic lymphocyst; A 10-year experience. Gynecol Oncol 29: 333-336, 1988.

17. Smith JW and Conway H: Selection of appropriate surgical procedures in lymphedema. Introduction of the hinged pedicle. Plast Reconstr Surg Transplant Bull 30: 10-31, 1962.

18. Abu-Rustum NR, Alektiar K, Iasonos A, Lev G, Sonoda Y, Aghajanian C, Chi DS and Barakat RR: The incidence of symptomatic lower extremity lymphedema following treatment of uterine corpus malignancies: A 12-year experience at Memorial Sloan-Kettering Cancer Center. Gynecol Oncol 103: 714-718, 2006.

19. Kuroda K, Yamamoto Y, Yanagisawa M, Kawata A, Akiba N, Suzuki K and Naritaka K: Risk factors and a prediction model for lower limb lymphedema following lymphadenectomy in gynecologic cancer: A hospital-based retrospective cohort study. BMC Womens Health 17: 50, 2017.

20. Ohba Y, Todo Y, Kobayashi N, Kaneuchi M, Watari H, Takeda M, Sudo S, Kudo M, Kato H and Sakuragi N: Risk factors for lower-limb lymphedema after surgery for cervical cancer. Int J Clin Oncol 16: 238-243, 2011.

21. Füller J, Guderian D, Köhler C, Schneider A and Wendt TG: Lymph edema of the lower extremities after lymphadenectomy and radiotherapy for cervical cancer. Strahlenther Onkol 184: 206-211, 2008

22. Mirandola D, Muraca MG, Sgambati E, Manetti M and Marini M: Role of a structured physical activity pathway in improving functional disability, pain and quality of life in a case of breast and gynecological cancer survivorship. J Clin Med 8: 531, 2019.

23. Rebegea L, Firescu D, Baciu G and Ciubara A: Psycho-oncology support. Brain (Bacau) 10: 77-88, 2019.

(i) $\odot$ This work is licensed under a Creative Commons Attribution-NonCommercial-NoDerivatives 4.0 International (CC BY-NC-ND 4.0) License. 